\title{
Deceased organ and tissue donation after medical assistance in dying and other conscious and competent donors: guidance for policy
}

\author{
James Downar MDCM MHSc, Sam D. Shemie MD, Clay Gillrie RN MSN, Marie-Chantal Fortin MD PhD, \\ Amber Appleby RN MM, Daniel Z. Buchman MSW PhD, Christen Shoesmith MD, Aviva Goldberg MD MA, \\ Vanessa Gruben LLM, Jehan Lalani MHA, Dirk Ysebaert MD PhD, Lindsay Wilson MHA, Michael D. Sharpe MD; \\ for Canadian Blood Services, the Canadian Critical Care Society, the Canadian Society of Transplantation and \\ the Canadian Association of Critical Care Nurses
}

Cite as: CMAJ 2019 June 3;191:E604-13. doi: 10.1503/cmaj.181648

See related article at www.cmaj.ca/lookup/doi/10.1503/cmaj.190352

CMAJ Podcasts: author interview at https://soundcloud.com/cmajpodcasts/181648-guide

I n Canada, organ donation from deceased donors is a common practice that saves or improves the lives of more than nearly 2000 Canadians every year, accounting for more than 3 of 4 of all transplanted organs. ${ }^{1}$ Deceased donation is permitted after either neurologic or circulatory determination of death, with the latter accounting for $25 \%$ of all organs donated in Canada in 2017. ${ }^{1}$ The current Canadian guideline recommendations for donation after circulatory determination of death, published in 2006, address the conventional scenario of an unconscious, incapable, critically ill patient not expected to survive the withdrawal of life-sustaining measures (WLSM). ${ }^{2}$

Two recent developments in Canada have led to a scenario not anticipated at the time of the 2006 guideline - requests for donation after circulatory determination of death from patients who are conscious and capable. The first development is the legalization of medical assistance in dying (MAiD), ${ }^{3-5}$ and the second, an anecdotal increase in requests for organ donation by patients with advanced neuromuscular diseases who have decided to have life-sustaining measures withdrawn. The ability of donors to give first-person consent for both MAiD or WLSM and organ donation creates emotional and moral challenges for health care professionals, and raises unprecedented ethical and practical challenges for patients, families, health care professionals and institutions, and society.

Prompted by requests from patients, Canadian practitioners requested guidance for policy development. In response, Canadian Blood Services worked in consultation with the Canadian Neurological Sciences Federation and in collaboration with the Canadian Critical Care Society, the Canadian Society of Transplantation and the Canadian Association of Critical Care Nurses to develop ethical, legal and clinical guidance for policies about

\section{KEY POINTS}

- First-person consent for organ donation after medical assistance in dying (MAiD) or withdrawal of life-sustaining measures (WLSM) should be an option in jurisdictions that allow MAiD or WLSM and donation after circulatory determination of death.

- The most important ethical concern - that the decision for MAiD or WLSM is being driven by a desire to donate organs should be managed by ensuring that any discussion about organ donation takes place only after the decision for MAiD or WLSM is made.

- If indications for MAiD change, this guidance for policies and the practice of organ donation after MAiD should be reviewed to ensure that the changes have not created new ethical or practical concerns.

managing deceased organ donation in conscious, competent donors. The full guidance document is available in Appendix 1, available at www.cmaj.ca/lookup/suppl/doi:10.1503/cmaj .181648/-/DC1. Here, we present key considerations and selected recommendations from the full guidance document.

\section{Scope}

The target audience of this guidance consists of clinicians, organ donation organizations, end-of-life care experts, MAiD providers and policy-makers. This document is intended to inform policies related to offering organ and tissue donation to patients who have made a decision that will lead to imminent death. These are conscious, competent patients who have chosen to withdraw mechanical ventilation, including invasive or noninvasive 
mechanical ventilation; conscious, competent patients who have chosen to withdraw extracorporeal support, including extracorporeal membrane oxygenation or other mechanical circulatory support; and eligible patients who have requested MAiD.

The issues that are out of scope for this guidance include the ethics of MAiD or WLSM, best practices for MAiD or WLSM independent of organ and tissue donation, donation by euthanasia (i.e., organ donation that does not adhere to the dead donor rule) and living organ donation.

\section{Methods}

Canadian Blood Services organized and funded the development of this guidance in response to requests from practitioners and policy-makers.

\section{Guidance panel}

Canadian Blood Services convened a panel of experts from relevant fields, including organ donation, ethics, law, intensive care, end-of-life care and neurology (S.S., M-C.F., D.B., C.S., A.G., D.Y. and V.G.). Members of the Canadian Blood Services Deceased Donation team also participated (C.G., A.A., J.L.). The panel was cochaired by J.D. and M.S.

\section{Development process}

Canadian Blood Services hosted a guidance development meeting in Toronto on May 15 and 16, 2017. Medical, legal and ethical experts; patients considering MAiD and organ donation; and an expert from Belgium participated (see full participant list in Appendix 1).

The objectives were to analyze organ and tissue donation in the conscious, competent patient from legal, medical and ethical perspectives; develop and disseminate guidance for policies and clinical procedures for offering organ and tissue donation to patients who have made a decision that will lead to imminent death (see Scope section); develop a knowledge translation strategy that includes all relevant stakeholders; and identify questions for research.

The guidance panel defined the scope, assumptions and key considerations of the guidance document and the workshop a priori (Appendix 1), based on the requests for guidance received by Canadian Blood Services. The guidance was developed via a process previously used for the national recommendations on organ donation after cardiocirculatory death. ${ }^{2}$

Before the workshop, the guideline panel commissioned a public opinion survey from Ipsos-Reid. Panel members searched for published studies and guidelines relevant to important topics, such as previous experience with organ donation in the conscious, competent adult (V.G., D.Y. and A.G.), patients with amyotrophic lateral sclerosis as organ donors (C.S.) and conscientious objection (D.B., V.G.), and developed background documents to guide and support discussion (Appendix 1). We provided these documents to all participants in advance of the workshop.

We structured the workshop around plenary presentations by Canadian and international clinicians, organ donation and transplantation ethicists and legal experts, a coroner and patient partners. The workshop included participants with a range of opinions about the practice of MAiD, and participants were free to share those opinions, but regardless of their support or opposition to MAiD, participants thought that if MAiD and organ donation were legal options, it was important to develop guidance to inform policies about them.

Participants were divided into smaller groups throughout the meeting to discuss and make recommendations regarding specific challenge questions that were informed by the background documents and expert presentations. Key points and conclusions from these groups were shared and discussed in plenary. Based on the material and notes from the small group and plenary discussions, the guidance panel developed and refined a series of recommendations meant to reflect the consensus of participants at the workshop. The guidance panel members agreed unanimously on the final guidance document presented here.

Given that this guidance was intended to address ethical and practical considerations relevant to policies and practice, the guidance panel did not think it was appropriate to assign a relative strength to any recommendation or rate the quality of evidence (similar to the previous guidance on donation after circulatory determination of death ${ }^{2}$ ). Some practical recommendations were informed by limited clinical experience and some unpublished data, but were not evidence based.

\section{Management of competing interests}

All members of the guidance panel declared any competing interests before any presentations to panel members while planning the meeting, and all presenters at the meeting declared their competing interests before their presentations.

To manage competing interests further, the scope of the guidance did not include a reference to any medications or devices, or any technical aspect of MAiD or donation after circulatory determination of death. Panel members, presenters and meeting participants were free to declare their personal views on MAiD or donation after circulatory determination of death but were not required to do so. All participants were from jurisdictions where MAiD and donation after circulatory determination of death are legal and practised.

\section{Recommendations}

Selected recommendations and key considerations are presented below. See Table 1 for a summary of all recommendations. More information on the evidence from the scoping reviews and discussion supporting each recommendation can be found in the full guidance document (Appendix 1).

\section{Deceased organ donation in conscious and competent patients}

Medically suitable, conscious and competent patients who provide first-person consent to end-of-life procedures should be given the opportunity to donate organs and tissues. Patients who seek MAiD or WLSM should not be prohibited from donating organs and tissue. 
Table 1 (part 1 of 3): Summary of recommendations

\section{Deceased organ donation in conscious and competent patients}

Medically suitable, conscious and competent patients who provide first-person consent to end-of-life procedures should be given the opportunity to donate organs and tissues. Patients who seek MAiD or WLSM should not be prohibited from donating organs and tissues.

Before consenting to WLSM or MAiD, patients should carefully consider all end-of-life options with their physician or health care professional.

\section{Referral to an organ donation organization}

Referral to the organ donation organization should occur as soon as is practical after the decision to proceed with WLSM or determination of eligibility for MAiD. Preliminary evaluation of the patient's eligibility to donate should be performed before the donation approach, if possible. This avoids the potential distress of making a request or obtaining consent for donation only to have to inform the patient that they are medically or logistically ineligible.

\section{Conversations about donation}

The decision to proceed with MAiD or WLSM must be separate from, and must precede, the decision to donate.

Treating physicians, MAiD providers and MAiD assessors should be educated on how to respond to inquiries concerning organ donation. This should include how the decision to donate may affect the end-of-life care process and options, and when to refer patients to the organ donation organization. The organ donation organizations should develop checklists or discussion guides to facilitate donation conversations to ensure patients are consistently well informed.

All eligible, medically suitable patients should be given an opportunity to consider organ and tissue donation, consistent with provincial or territorial required referral legislation, regional policy and ethical principles of respect for autonomy and self-determination. However, this must be reconciled with regional values and health care culture. Initially, some jurisdictions might prefer to begin with systems that respond only to patient-initiated requests.

Donation coordinators will have to tailor their conversations to ensure the patient remains the centre of the MAiD or WLSM and organ donation process, to ensure patient autonomy.

When an approach is to be made, discussions should happen early to allow individuals time to consider the options, ask questions and plan accordingly.

Patients and their families should be provided with standardized information resources, such as online material or pamphlets, to help guide responses to donation inquiries. The decision to proceed with MAiD or WLSM must precede discussions about donation.

\section{Consent}

The patient must have the ability to provide first-person consent to MAiD or WLSM as well as to organ and tissue donation.

Physicians, MAiD assessors, and WLSM or MAiD providers should be cognizant of the risk of coercion or undue influence on patients to donate their organs; however, the patient's altruistic intentions should not be discouraged.

Donation discussions must respect patient autonomy, and first-person consent should be obtained and upheld. Although it is welcomed and encouraged that family members are included in donation conversations, consent must be obtained from the patient and conversations should be focused on them.

The individual should be informed and understand that they may withdraw consent for MAiD or donation at any time, and that withdrawal of consent for donation does not affect their consent for, or access to, MAiD or WLSM.

The donation team should make every effort to resolve conflict, through dialogue, between the patient's expressed wishes to donate and a family's disagreement. First-person consent should direct all subsequent decisions unless consent was revoked.

If a conscious and competent patient provides first-person consent to donate after WLSM but subsequently loses decisional capacity, there is a strong case for proceeding with donation after WLSM because the patient was adequately informed about the decision by a trained donation expert and gave consent in the context of their illness and an anticipated imminent death. However, if a patient loses capacity before the MAiD procedure, then MAiD procedures cannot be carried out.

The donation team must understand and abide by the laws and policies of their jurisdiction with respect to reporting of MAiD deaths (e.g., coroner, special committee). To facilitate donation, these parties should be contacted before the MAiD procedure, in accordance with the current laws and policies.

\section{Donor testing and evaluation}

Primary care physicians, staff of organ donation organizations, MAiD providers and transplant teams should work to minimize the impact and inconvenience to the patient of donating their organs. This could include scheduling home visits for blood draws and coordinating investigations (e.g., $x$-rays, ultrasound) to minimize hospital visits and inconvenience to the individual.

Transplant teams and surgeons should work with the donation team to determine the minimum necessary investigations, to avoid the burden of excessive assessments and testing.

Donor teams should routinely discuss the potential impact of unanticipated results from the donor investigations, including previously undiagnosed infectious diseases, and their impact on public health reporting and contact tracing. 
Table 1 (part 2 of 3): Summary of recommendations

\section{MAiD procedures}

Consent for MAiD must be reaffirmed before the MAiD procedure. The health care team or MAiD provider should reaffirm consent before relocation to the hospital and before beginning any antemortem interventions for the purposes of facilitating donation. This may reduce the momentum of the donation process and reduce the potential for patients to feel pressured to continue with MAiD in the interest of ensuring organ donation.

\section{Determination of death}

The dead donor rule must always be respected. Vital organs can be procured only from a donor who is already deceased; the act of procurement cannot be the immediate cause of death.

For determination of death, absence of a palpable pulse alone is not sufficient. If arterial monitoring is not available, alternate means of determining absence of anterograde circulation should be used in conjunction with absence of a palpable pulse, such as a carotid perfusion ultrasound, Doppler monitoring, aortic valve ultrasound or an isoelectric electrocardiogram to determine asystole.

As with all cases of DCDD, death should be confirmed by a second physician after a 5-minute "no touch" period of continuous observation, during which time no donor-based interventions are permitted.

\section{Protection for patients}

\section{Separation of decisions}

To avoid any real or perceived conflict of interest, health care practitioners should separate the decision regarding WLSM or MAiD from discussions concerning donation. Providers who are assessing eligibility for MAiD should not be involved in donation discussions. Discussions concerning donation should happen only after WLSM decisions are made, or patients have been found eligible for MAiD by 2 independent assessments.

The primary health care team should acknowledge patient inquiries concerning donation that are made before a decision to proceed with MAiD or WLSM. General information on deceased organ and tissue donation may be provided. However, specific discussion and decisions pertaining to donation should wait until the decision to proceed with MAiD or WLSM has been finalized.

Patients may wish to postpone their MAiD procedure, owing to a temporary improvement in their health or an event they wish to experience before their death. The freedom of the patient to postpone their MAiD procedure must be reinforced and preserved, and every effort should be made to honour their wishes to donate their organs should their MAiD procedure be rescheduled.

\section{Directed and conditional donation}

No restrictions should be placed on potential organ recipients. Directed deceased donation (direction of a patient's organs to a specific recipient) or conditional donation (e.g., organs will be donated only if the patient can place conditions on what social groups may or may not access them) from patients considering MAiD or WLSM should be neither offered nor encouraged.

Living donation before death from patients considering MAiD or WLSM should be neither offered nor encouraged.

Should a patient insist on directed deceased donation or living donation before death, the request should be considered on a case-by-case basis.

\section{Separation of roles}

Consistent with current guidelines and practice regarding DCDD, separation should be maintained between the end-of-life care, donation and transplant teams. Surgical recovery and transplant teams should not be involved in the patient's end-of-life care or MAiD or WLSM procedure. The only exception is insofar as they may provide guidance for minimal requirements for donor investigations or premortem interventions.

Patients who wish to donate their organs after MAiD or WLSM, but who request that their decision to pursue MAiD or WLSM remain confidential, should be informed of the risk that their family members may discover incisions associated with surgical retrieval of organs. They should be encouraged to disclose their decision to family members; however, there is no obligation to stop the donation process should the patient wish to maintain the confidentiality of their MAiD or WLSM procedure.

That an organ donor received MAiD should not be disclosed to the potential recipient during allocation; however, medically relevant information regarding their underlying disease may be disclosed according to guidelines for exceptional distribution, where applicable.

\section{Supports for patients and families}

Specially trained professionals, such as donation physicians and coordinators, patient navigators or social workers, must be available to answer the patient's questions and facilitate the coordination of their MAiD or WLSM and donation. This may take place over a period of many weeks. The patient and their family must be provided with specific instructions on how to access these resources.

Support should be available in an optimally convenient location and setting for the patient, such as home visits or coordination with visits to clinics. For patients in remote locations, video-based technologies may be of assistance.

The donation team should work with the patient, their family and the MAiD or WLSM provider to develop a plan and best possible options for the MAiD or WLSM procedure that accommodates the wishes of the patient, preserving the opportunity to donate and reconciling coordination of hospital logistics.

Ongoing access to support for patients and their families is critical. Despite patient consent, donation might not proceed, owing to failure to find a suitable recipient, deterioration of health that compromises medical eligibility to donate, surgical findings during organ recovery, or withdrawal of consent by the patient. These patients and their families must continue to receive support even if donation does not proceed.

Continued support must be available to family members after the patient's death. Processes need to be developed to ensure families are given the opportunity to provide feedback on their experience, which may help with their grieving process and may help inform quality improvement measures. 
Table 1 (part 3 of 3): Summary of recommendations

\section{ALS and neurodegenerative diseases}

People with ALS and patients with other nontransmissible neurodegenerative diseases should be offered the opportunity to donate organs after their death.

Organ donation organizations should exercise caution regarding allocation of organs from donors with undiagnosed or rapidly progressive neurodegenerative diseases, as these may pose elevated risks to recipients. Organ allocation in this context should follow existing exceptional distribution policies and practices.

Transplant professionals must balance the benefits of the transplant against any potential for harm of receiving a transplant of an organ from a donor with a neurologic illness. Transplant professionals must use their discretion to help the transplant candidate navigate the decision. The surgeon may wish to consult the donor's neurologist to help inform their advice to the transplant candidate.

All cases of ALS or other neurodegenerative diseases that arise in transplant recipients should be reported to Health Canada, to determine potential associations with donor illness and the baseline risk of neurodegenerative illness in transplant recipients (e.g., whether transplant recipients, in general, have rates of ALS that differ from the general population).

Physicians who follow organ recipients should be aware that the donation was by a patient with neurodegenerative disease such as ALS; aware of theoretical transmission risk of neurodegenerative diseases; and cognizant of symptoms or complaints that warrant further investigation by a neurologist to determine if a neurodegenerative disease is present.

Active monitoring (i.e., regular visits to a neurologist) is not recommended for transplant recipients who have received an organ from a donor with a neurodegenerative disease. Neurological monitoring would impose a substantial burden on the recipient and present no benefit to the recipient, particularly as there is currently no value in early detection of these illnesses.

Information resources should be available for transplant candidates and for transplant professionals to help with the decision regarding whether to accept or refuse an organ for transplant. A means of obtaining a consult from a specialist neurologist in neurodegeneration may also be useful in helping the potential recipient make an informed decision. This information should also be available to organ donation organizations and the donation professionals responsible for assessing the eligibility of the patient who is considering donation.

\section{Health care professionals}

Health care professionals may exercise a conscientious objection to MAiD or WLSM specifically, but they should strive to accommodate the wishes of the donor by ensuring that their objection to MAiD or WLSM does not impede the ability of the patient to donate.

Health care professionals should act in accordance with provincial and territorial requirements as well as professional and regulatory college requirements for effective referral.

Health care professionals responsible for the care of conscious, competent patients who have requested WLSM or MAiD and donation should be briefed so they are familiar with the patient's end-of-life plan and relevant policies and procedures.

Debriefing after the procedure (i.e., MAiD or WLSM with or without donation) should be offered every time to all members of the health care team who participated. Debriefing by an external resource may be beneficial so that team members feel comfortable sharing their experience.

Psychological support, such as that offered through employee assistance plans, should be accessed when required. Staff of employee assistance plans may benefit from additional training and education regarding MAiD with or without donation to adequately meet the needs of these health care professionals.

Hospitals must ensure that staff are available who are willing and able to honour the patient's wishes to donate after their death or have an effective referral plan in place.

Participation of health care professionals in MAiD and in organ donation by patients who received MAiD should be voluntary, when possible, without interfering with the patient's access to care. The health care team should be well informed and well briefed so they understand the patient's wishes and the outcome they are working toward, as well as relevant policies and procedures.

\section{Reporting}

Clinicians must be aware of the reporting and documentation requirements for MAiD and WLSM and for donation in their jurisdiction.

Records pertaining to organ donation after MAiD, as well as donation and transplant outcomes, should be reported federally and be accessible to clinicians, researchers and administrators. Transplant outcomes should be easily cross-referenced with the underlying illness of the MAiD donor.

Note: $\mathrm{ALS}=$ amyotrophic lateral sclerosis, DCDD = donation after circulatory determination of death, MAiD = medical assistance in dying, WLSM = withdrawal of life-sustaining measures

Before consenting to WLSM or MAiD, patients should carefully consider all end-of-life options with their physician or health care professional.

Withdrawal of life-sustaining measures, MAiD and organ donation enjoy broad public support, both individually and in combination, and there is an existing legal and ethical framework for each in Canada (Appendix 1). Workshop participants identified potential problems that might arise when combining these practices (e.g., that the decision to donate organs might influence the decision to pursue MAiD), but these were felt to be manageable. Organ donation has taken place after WLSM and MAiD in a relatively small number of cases in Canada and other jurisdictions ${ }^{6-8}$ without any apparent negative effects. 


\section{Conversations about donation}

The decision to proceed with MAiD or WLSM must be separate from, and must precede, the decision to donate.

All eligible, medically suitable patients should be given an opportunity to consider organ and tissue donation, consistent with provincial or territorial required referral legislation, regional policy and ethical principles of respect for autonomy and self-determination. However, this must be reconciled with regional values and health care culture. Initially, some jurisdictions might prefer to begin with systems that respond only to patient-initiated requests.

The most important ethical concern that arises from the combination of MAiD or WLSM and organ donation is the possibility that the decision to proceed with MAID or WLSM might be driven by the desire to donate organs. ${ }^{7}$ Workshop participants felt that the best way to mitigate this risk is to ensure that organ donation discussions do not occur until after the decision is made regarding MAiD or WLSM. An additional safeguard would be to not offer organ donation in cases of MAiD or WLSM specifically, but respond only to organ donation requests initiated by patients themselves. However, participants were concerned that this could undermine a patient's autonomous right to donate their organs.

\section{Consent}

The patient must have the ability to provide first-person consent to MAiD or WLSM as well as to organ and tissue donation.

The individual should be informed and understand that they may withdraw consent for MAiD or donation at any time, and that withdrawal of consent for donation does not affect their consent for, or access to, MAiD or WLSM.

Although there are potential concerns with first-person consent for organ donation after circulatory determination of death (including the permissibility of treatments administered to the dying donor solely for the health of the donated organ), ${ }^{9}$ the informed, contemporaneous consent of a conscious and competent patient is the gold standard for decision-making in medicine..$^{10}$ Family concerns are important, and families must be supported throughout the donation, both for their own sake and to retain trust in the organ donation process. However, family refusals should not override persistent requests from capable patients to donate organs. ${ }^{11}$

\section{Donor testing and evaluation}

Primary care physicians, staff of organ donation organizations, MAiD providers and transplant teams should work to minimize the impact and inconvenience to the patient of donating their organs. This could include scheduling home visits for blood draws and coordinating investigations (e.g., $x$-rays, ultrasound) to minimize hospital visits and inconvenience to the individual.

Anecdotal experience from early cases suggested that the burdens of deceased organ donor testing may be substantial for patients on an outpatient basis, particularly when they have grievous and irremediable conditions and a limited life expectancy. Outpatient assessment of cardiac and pulmonary function should be reduced as much as possible to avoid losing donors, and home care should be engaged when appropriate. In some cases, donors may opt to donate fewer organs to limit the burden of testing.

\section{Determination of death}

The dead donor rule must always be respected. Vital organs can be procured only from a donor who is already deceased; the act of procurement cannot be the immediate cause of death.

For determination of death, absence of a palpable pulse alone is not sufficient. If arterial monitoring is not available, alternate means of determining absence of anterograde circulation should be used in conjunction with absence of a palpable pulse, such as a carotid perfusion ultrasound, Doppler monitoring, aortic valve ultrasound or an isoelectric electrocardiogram to determine asystole.

As with all cases of donation after circulatory determination of death, death should be confirmed by a second physician after a 5-minute "no touch" period of continuous observation, during which time no donor-based interventions are permitted.

Donors must be deceased according to accepted criteria before organ retrieval. ${ }^{2}$ Medical assistance in dying by vital organ donation is not an acceptable mechanism of MAiD. ${ }^{12}$ Because the usual invasive monitoring is not present during MAiD, a noninvasive approach may be needed to confirm circulatory death. ${ }^{13}$ The organ procurement process cannot be implicated in the death of the patient in any way.

\section{Protection for patients}

\section{Separation of decisions}

To avoid any real or perceived conflict of interest, health care practitioners should separate the decision regarding WLSM or MAiD from discussions concerning donation. Providers who are assessing eligibility for MAiD should not be involved in donation discussions.

The primary health care team should acknowledge patient inquiries concerning donation that are made before a decision to proceed with MAiD or WLSM. General information on deceased organ and tissue donation may be provided. However, specific discussion and decisions pertaining to donation should wait until the decision to proceed with MAiD or WLSM has been finalized.

\section{Separation of roles}

Consistent with current guidelines and practice regarding donation after circulatory determination of death, separation should be maintained between the end-of-life care, donation and transplant teams. Surgical recovery and transplant teams should not be involved in the patient's end-of-life care or MAiD or WLSM procedure. The only exception is insofar as they may provide guidance for minimal requirements for donor investigations or premortem interventions. 
That an organ donor received MAiD should not be disclosed to the potential recipient during allocation; however, medically relevant information regarding their underlying disease may be disclosed according to guidelines for exceptional distribution, where applicable.

Transplant teams should be separate from those providing endof-life care to the donor, to ensure there are no competing interests in terms of providing comfort care and ensuring the suitability of the organs for transplant. ${ }^{2}$ A guideline on WLSM is available and its recommendations can be applied regardless of whether the person is a potential organ donor or not. ${ }^{14}$

The circumstances of the donor's death should not be disclosed to the recipient in cases of MAiD or WLSM or any other donation situation. ${ }^{15}$ The donor's underlying medical condition may be relevant to the recipient and their transplant team, and can be disclosed under existing recommendations for exceptional distribution. ${ }^{16}$

\section{Amyotrophic lateral sclerosis and neurodegenerative diseases}

People with amyotrophic lateral sclerosis and patients with other nontransmissible neurodegenerative diseases should be offered the opportunity to donate organs after their death.

Organ donation organizations should exercise caution regarding allocation of organs from donors with undiagnosed or rapidly progressive neurodegenerative diseases, as these may pose elevated risks to recipients. Organ allocation in this context should follow existing exceptional distribution policies and practices.

All cases of amyotrophic lateral sclerosis or other neurodegenerative diseases that arise in transplant recipients should be reported to Health Canada, to determine potential associations with donor illness and the baseline risk of neurodegenerative illness in transplant recipients (e.g., whether transplant recipients, in general, have rates of amyotrophic lateral sclerosis that differ from the general population).

Physicians who follow organ recipients should be aware that the donation was by a patient with neurodegenerative disease such as amyotrophic lateral sclerosis, aware of theoretical transmission risk of neurodegenerative diseases, and cognizant of symptoms or complaints that warrant further investigation by a neurologist to determine if a neurodegenerative disease is present.

Many organ donation organizations do not accept organs from people with amyotrophic lateral sclerosis or other undiagnosed neurodegenerative conditions. Some authors have urged caution in this area, ${ }^{17}$ but people with amyotrophic lateral sclerosis have successfully donated organs after WLSM, ${ }^{18}$ and there appears to be no elevated risk of developing amyotrophic lateral sclerosis or many other neurodegenerative conditions after donation from selected persons with amyotrophic lateral sclerosis (Appendix 1). Because data are scarce about many neurodegenerative conditions, ongoing follow-up with the recipient is needed.
Decisions to use organs from donors with undiagnosed or rapidly progressive neurodegenerative conditions should be individualized based on risk and benefit because of the concern about transmissibility. Some organ transplants are primarily for improving quality of life or independence; others are clearly for survival.

\section{Conscientious objection}

Health care professionals may exercise a conscientious objection to MAiD or WLSM specifically, but they should strive to accommodate the wishes of the donor by ensuring that their objection to MAiD or WLSM does not impede the ability of the patient to donate.

Although most regulatory bodies in Canada have policies that govern conscientious objection, ${ }^{19}$ it is not clear how those policies apply to organ donation (see detailed explanation in Appendix 1). Conscientious objectors may not wish to participate in a process that facilitates MAiD or WLSM. But once the donor is deceased, it is difficult to apply the same framework to organ retrieval, transport or transplantation, as none of these acts facilitate MAiD or WLSM. Objectors may feel that participation in retrieval, transport or transplantation is a type of validation of how the organs became available. However, it is unclear why this logic would not apply to donors who died from suicide or homicide. Inconsistent application of conscientious objection policies may lead to inequitable allocation of organs $^{20}$ or place substantial burdens on some staff and institutions. ${ }^{21}$

\section{Implementation}

Offering the opportunity for conscious, competent patients to donate their organs after their death by WLSM or MAiD will affect institutions, health care professionals and society. An overview of the process is provided in Figure 1, but this practice will require mechanisms for oversight, data collection and reporting. Research on quality assurance and improvement to ensure this option for care is performed ethically and safely is also important.

Medical assistance in dying remains a controversial practice for many, and those who do not accept the practice of MAiD may not be able to apply this guidance. There are existing reporting mechanisms for MAiD $3,4,22,23$ and organ and tissue donation in all jurisdictions, but none for WLSM. Documentation and reporting mechanisms will need to be modified to ensure that key recommendations were followed, such as the requirement for the decision on MAiD or WLSM to precede the decision on donation, or the necessity for a registry to track the development of neurodegenerative disease in recipients.

There is no specific timeline to update this guidance for policy, but any changes to the eligibility criteria for MAiD (e.g., advance consent for MAiD for incapable patients) or the practice of donation after circulatory determination of death would require a review of this document to ensure that it covers any new ethical or practical concerns. 


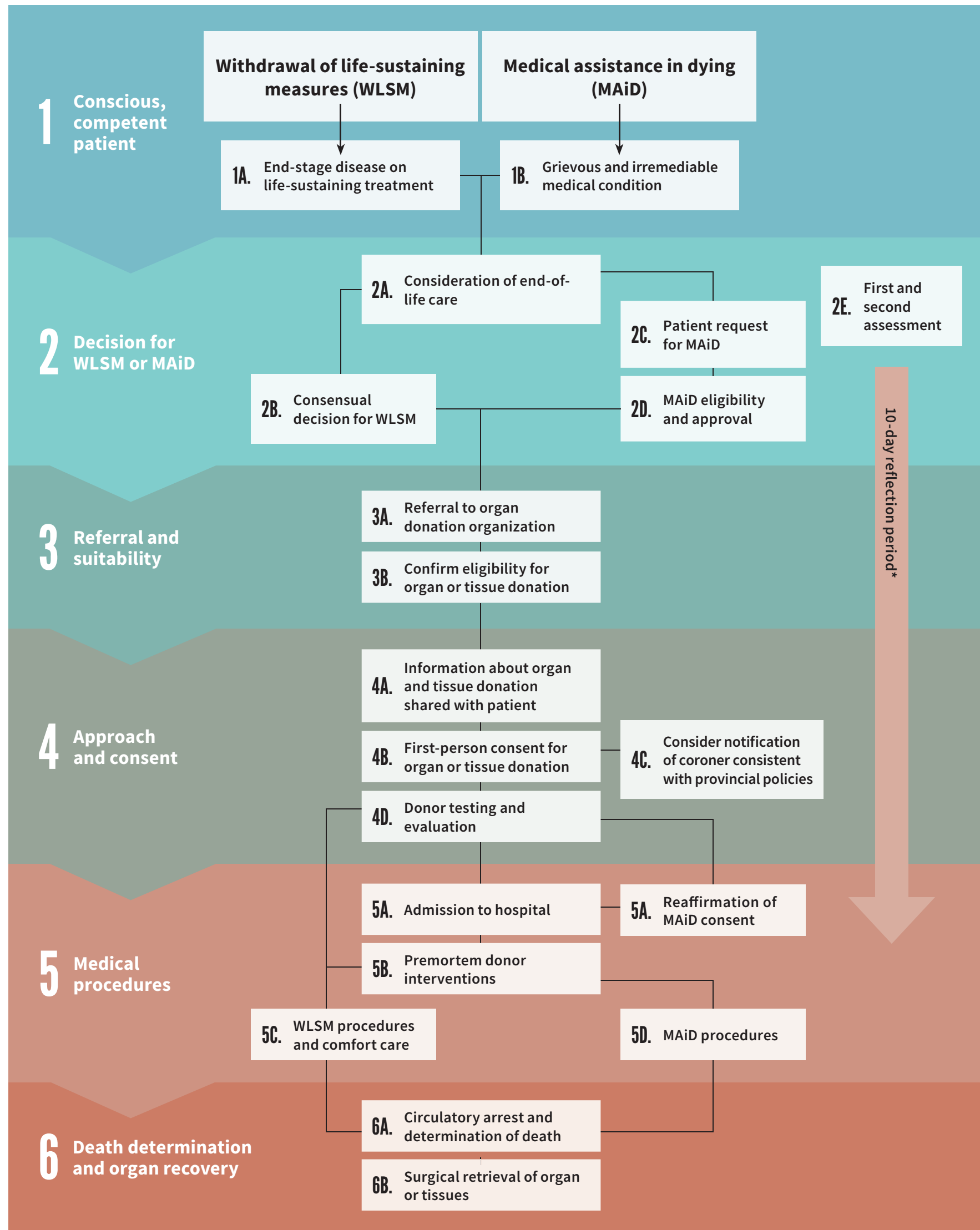

Figure 1: Clinical pathway for organ donation in conscious, competent patients in Canada. Note: MAiD = medical assistance in dying, WLSM = withdrawal of life-sustaining measures. ${ }^{*}$ The 10 -day reflection period begins from the day that the patient signs their written request, which should be after the first assessment of eligibility. This reflection period can be shortened if both assessors agree that the patient appears likely to die or lose capacity. 


\section{Other guidelines}

There are no previous published guidelines for policies and the practice of deceased organ and tissue donation for conscious, competent donors with respect to both MAiD and WLSM. There is a Dutch guideline for the practice of organ donation after euthanasia (but not WLSM), ${ }^{24}$ and manuals have been published in Canada ${ }^{25}$ and the Netherlands ${ }^{26}$ to help health care organizations develop a program for organ donation after MAiD. There are also Canadian guidelines for WLSM ${ }^{14}$ and donation after circulatory determination of death, ${ }^{2}$ but the latter does not assume that first-person consent is an option. There are no published guidelines regarding the advisability of organ donation from donors with neurodegenerative diseases such as amyotrophic lateral sclerosis, or the role of conscientious objection for organ donation in this context, and research is needed to inform practice in this area, as indicated in the present report.

\section{Gaps in knowledge}

Notably, directed deceased donation in conscious, competent patients is not recommended, although directed donation is permitted for living donors in many jurisdictions under specific circumstances. ${ }^{27}$ If a person was eligible for MAiD and had a relative or friend who was waiting for an organ, it would be difficult to exclude the possibility that the decision regarding organ donation had driven the request for MAiD, either to facilitate the donation process or to enhance the function of the transplanted organ.

A recent report of 2 Dutch cases of organ donation after MAiD initiated in the home ${ }^{28}$ raises the possibility of that practice occurring in Canada. Allowing the MAiD procedure to start outside the hospital would be more patient centred, and possibly improve the patient and family experience, but there are practical considerations that are not covered in this guidance (e.g., the logistics of transportation to hospital mid-procedure).

Finally, although some authors have suggested that organ donation after MAiD might have a substantial impact on organ availability, ${ }^{29,30}$ the practice of organ donation after MAiD in Canada is still rare. More data about this practice must be collected to determine whether this guidance document is helpful or needs to be updated.

\section{Conclusion}

In this report, we present guidance for policy and the practice of deceased organ donation in the conscious and competent donor. Given the relatively low incidence of MAiD and WLSM among people who are eligible to donate organs in Canada, this practice is unlikely to substantially affect the supply of organs for potential recipients on waiting lists. The purpose of this guidance is to inform the development of policies to help clinicians navigate the medical, legal and ethical challenges that arise when they respect a person's autonomous right to request MAiD or WLSM and organ donation.

Although many of these recommendations were informed by experience and data, areas of research remain, to ensure that a person's final wishes are honoured without placing their family, health care providers or potential recipients at risk of harm.

\section{References}

1. e-Statistics on organ transplants, waiting lists and donors. Ottawa: Canadian Institute for Health Information; 2017. Available: www.cihi.ca/ en/e-statistics-on-organ-transplants-waiting-lists-and-donors (accessed 2018 Dec. 10).

2. Shemie SD, Baker AJ, Knoll G, et al. National recommendations for donation after cardiocirculatory death in Canada: donation after cardiocirculatory death in Canada. CMAJ 2006;175:S1.

3. Carter v. Canada (Attorney General), 2015 SCC 5, [2015] 1 S.C.R. 331.2015 Feb. 6.

4. An act to amend the Criminal Code and to make related amendments to other acts (medical assistance in dying), 2016, Bill C-14. Assented June 17, 2016 (42nd Parliament, 1st session).

5. Bill no. 52: an Act respecting end-of-life care. Québec: National Assembly of Quebec; 2014.

6. Desschans B, Evrard P; Coordinator Transplant Section of the Belgian Transplant Society. Organ donation and transplantation statistics in Belgium for 2012 and 2013. Transplant Proc 2014;46:3124-6.

7. Allard J, Fortin MC. Organ donation after medical assistance in dying or cessation of life-sustaining treatment requested by conscious patients: the Canadian context. J Med Ethics 2017;43:601-5.

8. Bollen J, Ten Hoopen R, Ysebaert D, et al. Legal and ethical aspects of organ donation after euthanasia in Belgium and the Netherlands. J Med Ethics 2016;42: 486-9.

9. Overby KJ, Weinstein MS, Fiester A. Addressing consent issues in donation after circulatory determination of death. Am J Bioeth 2015;15:3-9.

10. Kirby J. Organ donation after assisted death: Is it more or less ethically-problematic than donation after circulatory death? Med Health Care Philos 2016;19:629-35.

11. Toews M, Caulfield T. Evaluating the "family veto" of consent for organ donation. CMAJ 2016;188:E436-7.

12. Wilkinson D, Savulescu J. Should we allow organ donation euthanasia? Alternatives for maximizing the number and quality of organs for transplantation. Bioethics 2012;26:32-48.

13. Wind J, van Mook WN, Dhanani S, et al. Determination of death after circulatory arrest by intensive care physicians: a survey of current practice in the Netherlands. J Crit Care 2016;31:2-6.

14. Downar J, Delaney JW, Hawryluck L, et al. Guidelines for the withdrawal of lifesustaining measures. Intensive Care Med 2016;42:1003-17.

15. Guidance for donor and recipient information sharing. Richmond (VA): Organ Procurement and Transplantation Network, United Network for Organ Sharing; 2012. Available: https://optn.transplant.hrsa.gov/resources/guidance/guidance -for-donor-and-recipient-information-sharing/ (accessed 2019 Apr. 16).

16. Safety of human cells, tissues and organs for transplantation regulations, SOR/2007-118 [consolidation]. Amended Feb. 11, 2015. Available: https://laws-lois .justice.gc.ca/PDF/SOR-2007-118.pdf (accessed 2019 Feb. 21).

17. Holmes BB, Diamond MI. Amyotrophic lateral sclerosis and organ donation: Is there risk of disease transmission? Ann Neurol 2012;72:832-6.

18. Smith TJ, Vota S, Patel S, et al. Organ donation after cardiac death from withdrawal of life support in patients with amyotrophic lateral sclerosis. J Palliat Med 2012;15:16-9.

19. Shaw J, Downie J. Welcome to the wild, wild north: conscientious objection policies governing Canada's medical, nursing, pharmacy, and dental professions. Bioethics 2014;28:33-46.

20. Savulescu J. Conscientious objection in medicine. BMJ 2006;332:294-7.

21. Fovargue S, Neal M. 'In good conscience': conscience-based exemptions and proper medical treatment. Med Law Rev 2015;23:221-41.

22. Smets T, Bilsen J, Cohen J, et al. The medical practice of euthanasia in Belgium and The Netherlands: legal notification, control and evaluation procedures. Health Policy 2009;90:181-7.

23. Emanuel EJ, Onwuteaka-Philipsen BD, Urwin JW, et al. Attitudes and practices of euthanasia and physician-assisted suicide in the United States, Canada, and Europe. JAMA 2016;316:79-90.

24. Mulder H, Olthuis $\mathrm{G}$, Siebelink M, et al. Guideline Organ donation following euthanasia [article in Dutch]. Ned Tijdschr Geneeskd 2017;161:D2135. 
25. Organ and tissue donation following medical assistance in dying: program development toolkit. Toronto: Trillium Gift of Life Network; 2018. Available: www.giftoflife.on.ca/resources/pdf/healthcare/MAID_Program_Development_ Toolkit_for_Hospitals_(Sept_10_2018).pdf (accessed 2018 Dec. 2).

26. Bollen J, de Jongh W, Hagenaars J, et al. Organ donation after euthanasia: a Dutch practical manual. Am J Transplant 2016;16:1967-72.

27. AMA Code of Medical Ethics. In: Chapter 6: Opinions on organ procurement \& transplantation. Chicago: American Medical Association; 2016. Available: www. ama-assn.org/sites/ama-assn.org/files/corp/media-browser/code-of-medical -ethics-chapter-6.pdf (accessed 2018 Dec. 2).
28. Mulder J, Sonneveld JPC. Organ donation after medical assistance in dying at home. CMAJ 2018;190:E1305-6.

29. Bollen J, van Smaalen T, Ten Hoopen R, et al. Potential number of organ donors after euthanasia in Belgium. JAMA 2017;317:1476-7.

30. Shaw DM. Organ donation after assisted suicide: a potential solution to the organ scarcity problem. Transplantation 2014;98:247-51.
Competing interests: Canadian Blood Services reports receiving grants from Health Canada and all provincial and territorial governments, during the conduct of the study. James Downar reports receiving personal fees from Boehringer-Ingelheim (Canada), Novartis and the Ontario College of Family Physicians, outside the submitted work. Dr. Downar is also the previous co-chair of the Physicians' Advisory Committee for Dying with Dignity Canada, an advocacy group for the legalization of medical assistance in dying in Canada. Sam Shemie reports receiving personal fees from Canadian Blood Services, during the conduct of the study. Vanessa Gruben reports receiving a grant from Canadian Blood Services, during the conduct of the study. Aviva Goldberg reports that as a nephrologist, she is primarily involved in the care of transplant recipients. No other competing interests were declared.

This article has been peer reviewed.

Affiliations: Division of Palliative Care, Department of Medicine (Downar), University of Ottawa, Ottawa, Ont.; Department of Critical Care (Downar), The Ottawa Hospital, Ottawa, Ont.; Department of Pediatrics (Shemie), McGill University, Montréal, Que.; Canadian Blood Services (Shemie, Gillrie, Appleby, Lalani, Wilson), Ottawa, Ont.; Bioethics Program (Fortin), Department of Social and Preventive Medicine, École de santé publique de l'Université de Montréal, Montréal, Que.; Dalla Lana School of Public Health (Buchman), University of Toronto, Toronto, Ont:; Department of Neurology (Shoesmith), Western University, London, Ont.; Department of Pediatrics and Child Health (Goldberg), University of Manitoba, Winnipeg, Man.; Faculty of Law (Gruben), University of Ottawa, Ottawa, Ont.; Faculty of Medicine (Ysebaert), University of Antwerp, Antwerp, Belgium; Department of Anesthesia and Perioperative Medicine (Sharpe), Schulich School of Medicine, Western University, London, Ont.
Guidance panel members: James Downar, Sam D. Shemie, Clay Gillrie, Marie-Chantal Fortin, Amber Appleby, Daniel Z. Buchman, Christen Shoesmith, Aviva Goldberg, Vanessa Gruben, Jehan Lalani, Dirk Ysebaert, Lindsay Wilson, Michael D. Sharpe.

Contributors: James Downar, Sam Shemie, Clay Gillrie, Amber Appleby, Jehan Lalani and Michael Sharpe conceived the guidance development project. All authors contributed to the design and conduct of the guidance development meeting, and the acquisition, analysis and interpretation of data. All authors drafted the manuscript, revised it critically for important intellectual content, gave final approval of the version to be published and agreed to be accountable for all aspects of the work.

Funding: The guidance development process was funded by Canadian Blood Services.

Acknowledgements: This guidance was developed on behalf of Canadian Blood Services in collaboration with the Canadian Critical Care Society, the Canadian Society of Transplantation, and the Canadian Association of Critical Care Nurses. This report is dedicated to Dr. Shelly Sarwal and Dr. Linda Panaro, who provided unique perspectives and thoughtful insights into the development of this guidance. Their knowledge and advocacy informed and guided the workshop process and has helped in the creation of this document, which will guide practice and inform policy development at the provincial and territorial and national level. The planning committee would also like to acknowledge all individuals who contemplate organ donation as part of their end-of-life care after making the decision for medical assistance in dying or withdrawal of life-sustaining measures.

Correspondence to: James Downar, jdownar@toh.ca 